\title{
Revisión de literatura: un acercamiento al aprendizaje autónomo de las lenguas extranjeras e interculturalidad a través del ABP
}

\section{Literature review: an approach to autonomous learning of foreign languages and interculturality through PBL}

\author{
Karolina Vargas Berra*
}

Recibido: 3 de junio de 2020 Aceptado: 30 de noviembre de 2020 Publicado: 31 de enero de 2021

To cite this article: Vargas Berra, K. (2021). Revisión de literatura: un acercamiento al aprendizaje autónomo de las lenguas extranjeras e interculturalidad a través del ABP. Márgenes, Revista de Educación de la Universidad de Málaga, 2 (1), 21-40

DOI: https://doi.org/10.24310/mgnmar.v2i1.9479

\section{RESUMEN}

El trabajo que se presenta forma parte de una investigación de posgrado sobre el aprendizaje de lenguas extranjeras en el nivel superior, dónde se destaca particularmente el proceso del desarrollo autónomo referente al aprendizaje de lenguas extranjeras tomando en cuenta el factor lingüístico e interculturalidad apoyado de la estrategia del Aprendizaje Basado en Problemas (ABP). El objetivo fundamental de esta revisión se centra en un análisis bibliométrico que permita establecer en la discusión de qué manera y con qué finalidad se han abordado las temáticas, tomando en cuenta sus objetivos, metodologías y resultados. A partir de ello, contrastar y dar cuenta de lo que se ha hecho y las posibilidades de indagación para acrecentar el acervo científico. La metodología empleada para esto es una revisión sistemática y analítica de distintas fuentes de información (artículos, tesis, conferencias) halladas en bases de datos, repositorios y revistas indexadas con el objetivo de sintetizarlas en un ejercicio de articulación. En la parte final se discuten los alcances de las investigaciones a partir de sus resultados y aportaciones y se plantean algunas consideraciones para su implementación, como el abordaje de la cultura en términos sociolingüísticos en distintos escenarios, el desarrollo de la autonomía como un proceso meta-cognitivo y los roles de los docentes y estudiantes en el proceso de enseñanza aprendizaje.

Palabras clave: interculturalidad; autonomía; competencia lingüística; lenguas extranjeras; aprendizaje basado en problemas

\section{ABSTRACT}

This work is part of a research on foreign languages learning in higher education, highlighting the process of autonomous development considering the linguistic and intercultural factor supported by Problem-Based Learning as an strategy. The fundamental aim of this review, focused on bibliometric analysis, is to establish how, what and for what purpose the topics have been addressed, taking into account their objectives, methodologies and results, to contrast and present what has been done and the possibilities of inquiry to increase the scientific heritage. The methodology used is a systematic and analytical review of different sources of information (papers, theses, and conferences) found in databases, repositories and indexed journals with the purpose of 
synthesizing them in a confluence exercise. In the final part, the scope of the researches is discussed, based on their results and contributions. Some considerations for their implementation are also raised such as the approach to culture in sociolinguistics terms in different settings, the development of autonomy as a meta-cognitive process and the roles of teachers and students in the teaching-learning process.

Keywords: interculturality; autonomy; linguistics competence; foreign languages; problem-based learning

\section{INTRODUCCIÓN}

La enseñanza de lenguas extranjeras es una actividad que se ha practicado a lo largo de los años, primero de una manera informal para lograr la comunicación entre distintos pueblos y establecer alianzas comerciales o estratégicas, posteriormente, de una manera más formal estableciendo una determinada metodología y estrategias que han variado según las épocas (Martín, 2010). En la actualidad hay un principal énfasis en el enfoque comunicativo desde una perspectiva basada en la acción, por lo que se espera que el aprendiz realice tareas que lo lleven a deducir las reglas gramaticales, a desarrollar el pensamiento crítico, construir conocimientos y tomar decisiones en contextos culturales determinados.

Este trabajo pretende llevar a cabo un análisis bibliométrico a partir de la revisión de literatura sobre el aprendizaje autónomo de las lenguas extranjeras, la interculturalidad y el Aprendizaje Basado en Problemas (ABP) para establecer una discusión sobre la manera en que se ha abordado la temática en distintos contextos y con distintas finalidades. Cabe señalar que en todos los estudios revisados la vinculación entre los elementos se da considerando al ABP como la estrategia para la enseñanza de lenguas y mediante el cual se desarrolla los aspectos lingüísticocomunicativos y los interculturales.

El índice expositivo se inicia con un recorrido conceptual sobre la interculturalidad y su relación directa con la enseñanza de lenguas extranjeras, explicado las variaciones existentes. En seguida se trata la conceptualización del aprendizaje autónomo vinculado con la lingüística. Luego se presenta la estrategia del ABP de manera general y cómo ha sido empleada para la enseñanza de las lenguas extranjeras. Posteriormente se explica la metodología propuesta por Jiménez-Vásquez (2014) utilizada para llevar a cabo el análisis bibliométrico, enfatizando el proceso de búsqueda y sistematización. A continuación, se presentan resultados cuantificables, es decir, la estadística resultante de la búsqueda. En el penúltimo apartado se lleva a cabo una discusión donde se abordan las valoraciones de los estudios analizados mediante contraste y vinculación. Finalmente se presenta una conclusión general de la temática.

\section{LA INTERCULTURALIDAD EN LAS LENGUAS EXTRANJERAS}

El aspecto cultural es un elemento indisociable en el contexto de enseñanza de lenguas extranjeras. Es a través de él que se logran afianzar los referentes pragmáticos y sociolingüísticos que permiten una mejor comunicación en distintos contextos o situaciones. Abdellaouy (2018), Bizarro (2018) y Puren (2014) coinciden en que estos dos elementos, cultura y lengua, están íntimamente vinculados, coexisten y se nutren uno al otro. 
En la literatura se observa el término interculturalidad o educación intercultural relacionado a los idiomas y la cultura relacionada a las prácticas y modos de vida de una sociedad, de ahí que este último vocablo sea el más general. Cuando es descontextualizada del aprendizaje de lenguas extranjeras, la interculturalidad se sitúa en las relaciones sociales que se dan entre distintos grupos de distintas culturas, siendo la integración social y la lingüística; sus elementos clave.

Siguiendo esta línea, la interculturalidad en el contexto latinoamericano y desde la visión de los pueblos indígenas surge como una opción política y ética basada en la homogeneización, apostando por una educación para todos, popular y crítica. En este sentido Orozco-López argumenta que "el concepto educación intercultural es retomado por intelectuales indios nutridos del debate latinoamericano, al paso del tiempo, la educación intercultural derivó en una noción llamada funcional que perdió todo sentido político y se centró en aspectos de tolerancia, convivencia y respeto entre pueblos indígenas y la sociedad nacional mestiza” (2018, p.42). Como él, otros autores latinoamericanos como Herrera y Ortiz (2018) han abonado a los estudios sobre la interculturalidad desde esta perspectiva, no obstante, la revisión de literatura que atañe a esta investigación sólo considera los estudios interculturales en las lenguas extranjeras.

Retomando el planteamiento inicial, Pretceille (2011) realizó un estudio que aborda de manera crítica la pedagogía intercultural, refiriéndose a ella cómo una práctica en donde se lleva a cabo el aprendizaje de las culturas. Este aprendizaje es, la mayoría de las veces, colocado en segundo plano o es inexistente en las clases e incluso en los diseños curriculares y los syllabus de las asignaturas, priorizando los temas gramaticales. En el tesauro de la UNESCO se observan alternativas al término intercultural y conceptos relacionados. Se tiene así multiculturalidad, transculturalidad y biculturalidad, aculturación, identidad cultural, interacción, migración, socialización y contracultura (UNESCO, 2020).

El término y sus variantes pueden tener múltiples representaciones, como lo podemos constatar a continuación, y estas se deben principalmente a las ópticas desde donde se observen. Puren (2014) propone cinco representaciones comunes presentes en la didáctica de lenguas:

a. Transculturalidad: es considerada como un valor universal que tiene por finalidad reconocer los aspectos en común, se caracteriza por desarrollarse con una metodología tradicional.

b. La meta-culturalidad: se relaciona con los conocimientos culturales y la capacidad de hablar sobre ellos, la metodología en la que se basa es el aprendizaje activo.

c. La pluriculturalidad: está relacionada con actitudes y comportamientos que proveen las capacidades para vivir de manera armoniosa en una sociedad con culturas diversas; la metodología con la que se relaciona es la didáctica del plurilingüismo.

d. La co-culturalidad: se basa en un enfoque comunicativo desde una perspectiva accional y se relaciona con las concepciones y valores contextuales; se considera como la eficacia de interactuar y adoptar una cultura compartida.

e. La interculturalidad: es la capacidad para identificar aquellas cosas que no se comprenden en los primeros contactos con la lengua y cultura extranjera y que se relacionan con la propia. Su característica principal es la representación y su metodología más representativa es el enfoque comunicativo. 


\section{E S T U D O S Y E N A Y O S}

Piteira (2014) coincide con este autor y además añade el concepto de aculturación, visto como la integración social y afectiva (psicológica) en la que el estudiante asume la cultura del otro. Dicho concepto es recuperado del modelo para la adquisición de la segunda lengua desarrollado por Schumann y donde expone que cuanto más se asimila la cultura de la lengua mayor será el proceso de adquisición (Zaker 2016). A este entendido, cabe señalar que, se requiere de un ambiente propicio para que se genere la aculturación, misma que es observable en las zonas fronterizas, como la existente entre México y Estados Unidos; o en países donde la presencia de migrantes es alta, como los pertenecientes a la Unión Europea.

No obstante, estos no han sido los únicos autores que han tratado de dar luz al término, sus acepciones, usos e implicaciones en el proceso de aprendizaje de lenguas extranjeras. Abdellaouy (2018), Bizarro (2018), Doria (2017), Herrera y Ortiz (2018), Moya-Chávez, MorenoGarcía y Núñez-Camacho (2019); y Sumonte et al. (2019), por mencionar a los más recurrentes, han estudiado este fenómeno como un elemento formativo y de dinámica identitaria donde la comprensión del otro es necesaria para establecer interacciones efectivas. En este mismo tenor, Puren (2014) sugiere que toda formación social tiene como principio la concepción del otro, de esta manera se le puede comprender y al hacerlo generar vínculos que permitan la comunicación. Estas afirmaciones refuerzan el argumento de contar con una enseñanza de la cultura, logrando así una competencia intercultural que permite al estudiante aprender el idioma, así mismo comprender las pautas sociales en las que este es utilizado y cómo determina y nutre a la identidad de los miembros de un grupo social.

Retomando las variaciones del tesauro, y en relación con lo anterior, Hall y du Gay (2003) estudian la identidad cultural, indicando que es una temática que ha crecido y fortalecido con el paso de los años considerando la importancia de contar con una conceptualización del ser y del otro, permitiendo así una mayor consciencia, lo que llevará a la formación de la identidad $y$, en consecuencia, de la sociedad. Es por dicha razón que desde el inicio se debe tener clara la tipología de cultura que se pretende abordar en clase (Bizarro 2018), es decir, a partir de dónde se hará el abordaje: contenidos temáticos, expresiones comunes, formas de vida, cuestiones relacionadas con la familia, salud, educación, economía, etc.; con que metodología de enseñanza y el tipo de actividades que reforzarán dicho aprendizaje.

En esta exposición conceptual se puede decir que la interculturalidad es un aspecto relevante y desasociable en el aprendizaje de las lenguas extranjeras. Así mismo se observa que nutre de manera significativa la formación del estudiante, pasando por aspectos lingüísticos y gramaticales, para llegar a la comprensión del otro, en un nivel macro refiriendo a las culturas que se están descubriendo y en un nivel micro a la propia cultura y sus diferencias.

Ahora bien, al momento de ejecutar la interculturalidad mediante estrategias de enseñanza, la literatura refiere una aproximación en el aprendizaje activo desde una perspectiva accional o basada en tareas.

\section{EL APRENDIZAJE AUTÓNOMO Y LA LINGÜÍSTICA}

Para hablar de la autonomía lingüística es necesario primero disociar los términos de manera analítica. Por un lado, autonomía y por otro, lingüística. La autonomía guarda una semejan- 
za con la competencia autónoma, el aprendizaje auto-dirigido y la auto-regulación. Así mismo se relaciona con reflexión, aprendizaje, enseñanza, educación superior, universitarios, empleo, profesión, didáctica, gestión, objetivos, evaluación, socialización, meta-cognición, autorregulación y competencia (UNESCO, 2020). No obstante, al hablar de la autonomía dentro de las aulas, los términos acotados al mundo laboral son poco pertinentes.

La autonomía es considerada como un dominio clave en la gestión del aprendizaje, ya que es a través de ella que se puede alcanzar la independencia permitiendo el desarrollo del pensamiento crítico y, en consecuencia, la toma de decisiones. Por lo que se sostiene que la autonomía lleva al estudiante a poner en práctica su meta-cognición. Es una manera en que el individuo puede tener una mejor organización de pensamiento y lograr mejores reacciones ante situaciones inesperadas.

En este sentido, varios autores (Bravo-Cedeño, Loor-Rivadeneira y Saldarriega-Sambrano, 2017; Flores y Meléndez, 2017; Rivadeneira y Bustillos, 2017; Velázquez, Rodríguez y Nieves, 2018; Velázquez y Santiesteban, 2019) coinciden que es justo en la formación de la educación superior que la autonomía adquiere un valor inigualable, pues permite una satisfacción personal en los procesos de aprendizaje a través de la gestión del conocimiento, el refuerzo y retroalimentación de los contenidos temáticos vistos en clases. Debido a estas características se puede considerar a la autonomía como en elemento del aprendizaje activo centrado en el estudiante que articula, mediante la cognición, saberes previos, saberes nuevos, reestructuración, construcción y reconstrucción de conocimientos, así mismo, la capacidad y la habilidad de recuperarlos para emplearlos en situaciones determinadas y similares a las que fueron aprendidos.

En cuanto a la lingüística implica poner en acción una serie de aspectos que permiten la comunicación. Así mismo, el vocablo encierra a su vez sus componentes: fonética, gramática, ortografía, semántica, pragmática, sociolingüística, por mencionar los más recurrentes; los cuales son considerados conceptos específicos o relacionados entre sí según el Tesauro de la UNESCO (2020). Cuando en Marco Común Europeo de Referencia para las Lenguas (MCERL) hace referencia a este concepto, lo trata como una sub-competencia perteneciente al campo comunicativo, junto a la sociolingüística y la pragmática (Consejo de Europa, 2002).

De manera particular el MCERL aborda a la competencia lingüística como los conocimientos y habilidades relacionados con la pronunciación (fonología), el vocabulario (léxico), las reglas y/o normas (gramática) y la estructura de la frase (sintaxis). Su principal característica es el uso de estos conocimientos mediante el reconocimiento, recuperación y disponibilidad para poder ser usada en algún contexto en particular. Por lo que los aspectos cognitivos y meta-cognitivos están presentes.

A la par de la competencia lingüística encontramos a la pragmática que toma en cuenta el funcionamiento del código lingüístico, es decir, los actos del habla. En esta competencia se visualiza el dominio del discurso, por lo que aspectos culturales también están presentes; por ejemplo, reconocer la ironía o el humor. Si bien pareciera que la pragmática es la respuesta a las investigaciones en donde se tratan aspectos lingüísticos, culturales y autónomos, vale la pena estudiarlos de manera independiente. 
Ahora bien, a partir de la comprensión de los términos de manera aislada ya es posible articularlos y proponer la conceptualización de la autonomía lingüística como el proceso de aprendizaje de lenguas extranjeras que tiene el estudiante para identificar, recuperar y construir, de manera independiente, a partir de conceptos gramaticales discursos orales y escritos que le permitan la comunicación con los demás. Aunado a esto y retomando el primer apartado, los aspectos interculturales enriquecen dichos discursos, pues es en la pragmática donde se observa la eficacia de la competencia comunicativa e intercultural. Así mismo, una estrategia como el ABP es capaz de recuperar el desarrollo de la autonomía y explotar los elementos lingüísticos e interculturales de las lenguas extranjeras.

\section{EL APRENDIZAJE BASADO EN PROBLEMAS Y LA ENSEÑANZA DE LENGUAS EXTRANJERAS}

El ABP es una estrategia que se ha empleado, en su mayoría en la educación superior, a lo largo de los últimos 40 años. Su proceso de aplicación se basa en la investigación, análisis, toma de decisiones y resolución de problemas. Esto permite un mayor aprendizaje autónomo ya que "the students are expected to learn from the worlds knowledge and accumulated expertise by virtue of their own study and research [...] During this self-directed learning, students work together, discussing, comparing, reviewing, and debating what they have learned ${ }^{1}$ (Barrows, 1996, p. 6). De manera general, Muñoz (2017) menciona que el ABP contribuye al desarrollo académico y trabajo de habilidades donde se requiere de estudio independiente y autonomía, así como de capacidades colaborativas. En este sentido el ABP encierra múltiples posibilidades para ser utilizado en el aula pues considera el trabajo colaborativo, la auto-evaluación y la co-evaluación, la discusión, el rol del docente como guía y el rol de estudiante como responsable de sus aprendizajes; todo esto traducido en acciones presentes en la formación integral de los estudiantes, particularmente en la educación superior.

El ABP nació en los años setenta en un entorno de ciencias de la salud y, en su origen, era vista como una estrategia para aprender a resolver casos clínicos y atención al paciente. Se implementó para dar respuesta a las modificaciones curriculares de la Facultad de Ciencias Médicas de la Universidad de McMaster con la finalidad de preparar a los estudiantes de manera completa para satisfacer las demandas laborales (Morales y Landa, 2004). Su éxito permitió la expansión a otras universidades a nivel mundial.

Posteriormente en los años ochenta, debido a los resultados positivos que se observaban, otras disciplinas lo adoptaron y adaptaron para lograr aprendizajes más apegados a la realidad de los futuros profesionales (López, 2008). Como ejemplo se encuentran áreas de estudio como arquitectura, administración, economía, ingeniería, leyes y otras disciplinas de la educación, incluso bachillerato.

En el campo de las lenguas extranjeras conoce su aplicación a partir del nuevo milenio, como un medio para fortalecer el aprendizaje de idiomas, sistematización de reglas gramaticales y,

1 "se espera que los estudiantes aprendan a partir del conocimiento del mundo y las experiencias acumuladas por medio de su propio estudio e investigaciones. Durante este aprendizaje auto-dirigido los estudiantes trabajan de manera conjunta, discuten, comparan, revisan y debaten lo que han encontrado". Traducción propia. 
particularmente, desarrollar habilidades de producción y expresión oral y escrita; por lo que su pertinencia en esta investigación está presente y justificada. En la literatura se observa que Asia es el continente que más ha explotado este medio y ha realizado investigaciones sobre él, principalmente en países como Malasia, Indonesia y Tailandia. En estos estudios se rescatan dos corrientes: una es el rol del docente y del estudiante; y otra, la explotación de la estrategia; mismas que se exponen brevemente a continuación.

\section{METODOLOGÍA}

La metodología empleada está basada en la propuesta de Jiménez-Vásquez (2009) para elaborar estados del arte, misma que refiere tres etapas trianguladas que se articulan entre sí: la heurística, la hermenéutica y la holística. La autora refiere que en la primera etapa se tienen los acercamientos a las fuentes, la segunda permite analizar cada una de las partes desmenuzando la información recabada; $y$, finalmente, la tercera es la que permite sintetizar lo recuperado de manera puntual, estableciendo convergencias y divergencias que permiten encontrar los vacíos o vetas de los estudios. En este ejercicio se transita entre las distintas etapas nutriéndose constantemente para determinar un objeto de estudio que tome en cuenta aquellas variables que son posibles explorarse desde otras perspectivas, o bien, que se encuentran en terrenos fértiles para su exploración.

Esta metodología permite llevar a cabo un análisis bibliométrico a partir de las variables: ABP, autonomía, lingüística, interculturalidad y enseñanza-aprendizaje de las lenguas extranjeras. Estas son abordadas de manera independiente y se articulan en el apartado de discusión.

\subsection{Búsquedas}

La búsqueda de la información se realizó en distintos medios como documentos físicos, electrónicos, visuales, audios y conferencias. En dichas fuentes se hace acopio del máximo de información. Para ello se toma en cuenta las interrogantes ¿qué?, ¿dónde?, ¿para qué? y ¿cómo se quiere investigar? (Morel y Buitrargo, 2017).

Ahora bien, las indagaciones y búsquedas fueron realizadas de dos maneras distintas, una in situ, es decir, directamente en bibliotecas universitarias del estado de Puebla y Tlaxcala; y la otra mediante bibliotecas virtuales y herramientas de internet, siendo aquí donde se encontraron resultados fructíferos. De manera particular se indagó en EBSCO, Redalyc, Scielo, Springer, Scopus, Sci.hub, Dialnet, Elsevier como fuentes principales ya que se caracterizan por contar con una mayor rigurosidad y especialización, aunque también se recurrió directamente a las Revistas como el caso de Synergies, European Journal of Applied Linguistics, Opuntia Brava, RED, por mencionar las más frecuentes.

Las búsquedas se hicieron en torno a los conceptos "interculturalidad”, "autonomía”, "competencia lingüística” y “Metodología en la Enseñanza de Lenguas Extranjeras” a través de las opciones avanzadas de campo título y palabras clave usando los operadores boleanos OR, AND y NOT y los Tesauros de la UNESCO para precisar las búsquedas. 


\section{ESTUDIOS Y ENSAYOS}

En los primeros acercamientos de distinguen conceptos interrelacionados. Cultura, multiculturalidad, pluriculturalidad y bilingüismo se relacionan directamente con interculturalidad. Este vínculo, permite por un lado brindar un panorama más amplio de la conceptualización de la interculturalidad, y por otro lado acotar la búsqueda eliminando el bilingüismo, referido a espacios donde la lengua extranjera se emplea como un medio para tratar contenidos.

En cuanto al concepto de autonomía, este tiene semejanza con competencia autónoma, auto-aprendizaje, aprendizaje auto-dirigido y auto-regulación. Para fines del aprendizaje de las lenguas extranjeras se acota la búsqueda con los dos primeros términos ya que figuran en el MCERL, documento eje para estas prácticas. Finalmente, con respecto a la competencia lingüística, se identifica una correlación con los de competencia comunicativa, habilidades productivas (expresión oral y escrita) y receptivas (comprensión oral y escrita), así como con la lengua. (Ver Ilustración 1).

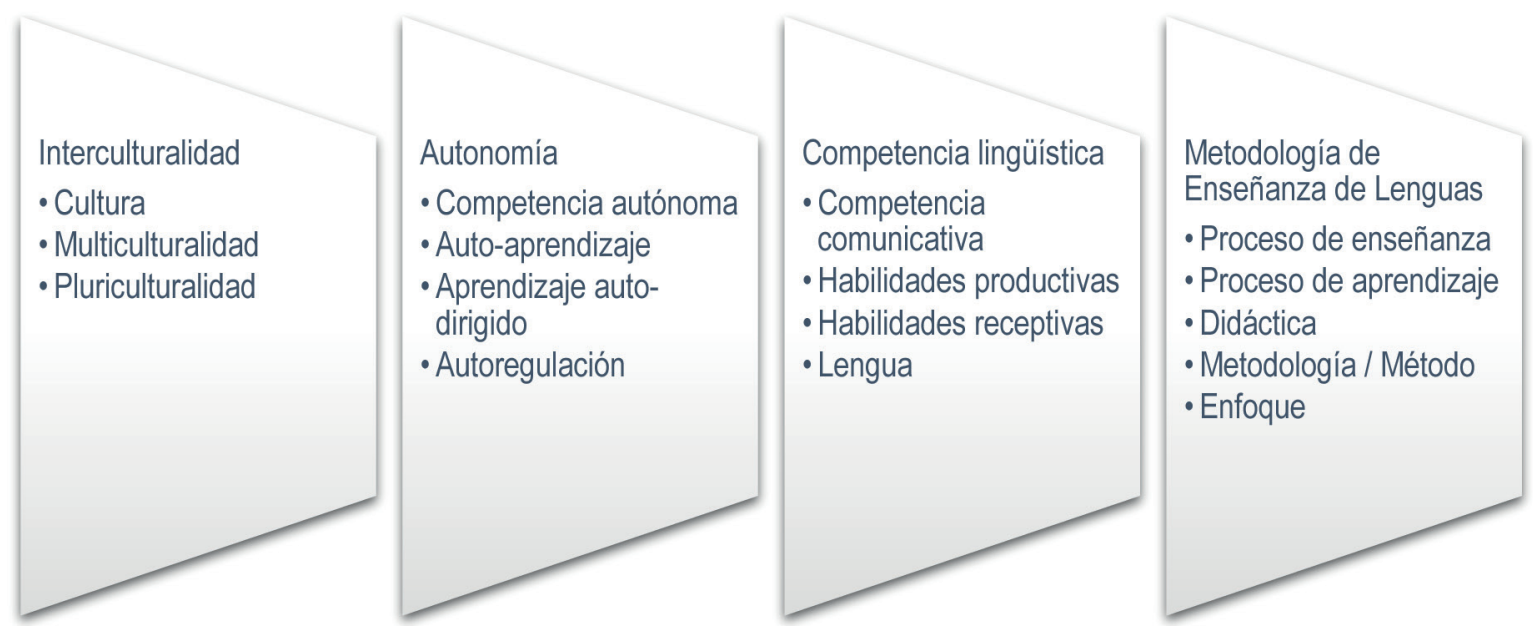

Ilustración 1. Conceptos centrales. Nota: ilustración de elaboración propia

Para completar las búsquedas, las palabras clave se ingresaron con su traducción en inglés (culture, interculturation, acculturation, autonomy, self-learning, linguistics, competence, approach, learning, teaching) y francés (culture, interculturalité, multiculturalisme, autonomie, compétence linguistique, linguistique sociolinguistique, procès d'enseginenment-apprentissage, aproche, méthode), lo que permitió un mayor número de información recabada.

\subsection{Sistematización de la información}

Una vez realizada la recuperación de información es necesario gestionarla para evitar fugas de material y también para administrar las fuentes de consulta y los análisis que se hacen a partir de las lecturas superficiales y profundas de cada una de ellas. Jiménez-Vásquez (2009) menciona que cada documento debe leerse, analizarse y clasificarse de acuerdo a su importancia. Así mismo, para concentrar la información recabada, se utilizaron dos recursos: una matriz en hoja de cálculo y un gestor de información, ambos permitieron organizar las fuentes para un acceso más eficaz y rápido. 


\section{ESTUDIOS Y ENSAYOS}

La matriz, también denominada tabla bibliográfica o de contenido, posibilita avanzar en el análisis de un texto o en la integración de varios textos y refleja la complejidad, la comparación y el contraste de información; y da cuenta de un acercamiento cuantitativo de la información (Jiménez-Vásquez, 2014). Es por ello que se considera como un medio efectivo de concentrar la información de manera esquemática.

Como puede apreciarse en la tabla 1, la matriz está conformada por 5 grandes campos: datos bibliográficos, campo temático, campo teórico, campo metodológico y ubicación; y al interior de ellos con 31 descriptores. La razón fundamental para establecer esta categoría es para facilitar la esquematización y control de la información, y a su vez, reconocer de manera efectiva los elementos que se quieren contrastar para observar las convergencias y divergencias de cada autor. Estos elementos son tomados de la propuesta de Esquivel (2013) y mediante su análisis y síntesis, permiten plantear nuevas preguntas.

Tabla 1. Matriz de referencia

\begin{tabular}{|c|c|c|c|c|}
\hline Datos bibliográficos & Campo temático & Campo teórico & Campo metodológico & Ubicación \\
\hline Autor(es) & $\begin{array}{l}\text { Dimensión de } \\
\text { estudio / Núcleo }\end{array}$ & \multirow{3}{*}{$\begin{array}{l}\text { Teoría o corriente } \\
\text { de pensamiento }\end{array}$} & Metodología & Ubicación USB \\
\hline Contacto & temático & & Enfoque & Liga \\
\hline Título del documento & Palabras clave & & Instrumentos & Liga alternativa \\
\hline Año de publicación & Resumen / Abstract & $\begin{array}{c}\text { Conclusiones del } \\
\text { autor }\end{array}$ & Participantes / Muestra & Mapa conceptual \\
\hline Editorial / Editor & Relevancia & Autores citados & Hipótesis / Preguntas de & Ubicación respaldo \\
\hline Ciudad de la investigación & Tema del & & & \\
\hline País de la investigación & documento & & & \\
\hline Referencia bibliográfica & Aspectos relevantes & & & \\
\hline (APA) & & & & \\
\hline Fecha de recuperación & & & & \\
\hline Tipo de publicación & & & & \\
\hline
\end{tabular}

Nota: tabla de elaboración propia

Como puede apreciarse en la Tabla 1, los datos bibliográficos consideran la información básica relacionada con la fuente de información. En orden descendente se observa primero al autor o autores, enseguida el contacto, este rubro no siempre es posible tenerlo, la intención de colocarlo es poder tener un acercamiento directo con el autor para resolver dudas puntuales. Posteriormente título del documento, año de publicación, editorial o editor, estos permiten remitir el documento o buscar más información en editoriales o revistas editoras con la finalidad de poder contrastar y encontrar más información. Luego se considera al país de la investigación, está razón es para determinar que temáticas son trabajadas y divulgadas en el globo. Finalmente, la fecha de recuperación y el tipo de publicación son colocados como consideración para la vigencia del documento. 
En la segunda columna se considera el campo temático. Este está integrado por la dimensión del estudio o núcleo temático, palabras clave y tema del documento, esta información sirve para conjuntar los documentos que presentan una misma temática y su contraste sea más rápido de evidenciar. Enseguida se ubica el resumen provistos por los documentos (en caso que lo tenga como en los artículos) este permite tener una visión global de la investigación reportada. Finalmente, en cuanto a la relevancia y aspectos relevantes se considera el valor de los estudios y los aspectos más significativos que pueden recuperarse para robustecer la investigación en curso.

En la tercera columna se considera el campo teórico. Esta sección sirve para identificar las posturas teóricas en las que se basan los estudios revisados, así como las conclusiones del autor y los autores citados. La importancia radica en conocer los marcos referencias sobre la teoría y quienes los están desarrollando, así se puede valorar su inclusión o exclusión dentro de esta investigación.

En la cuarta columna, se recupera el campo metodológico, mismo que se entrelaza con el campo teórico, en él se establecen los diversos diseños de investigación, los tipos de participantes, el tipo de preguntas de investigación, y los instrumentos. La intención de identificar estos elementos y saber la tendencia y las posibilidades que se tienen para el acercamiento de la temática.

Al final se halla la columna de ubicación, en ella se coloca el sitio donde se tienen almacenado el documento y tener un acceso más rápido. De igual manera se tiene la liga de donde se recupera y finalmente un mapa conceptual que ayuda a esquematizar la información relevante de cada documento. Esto permite una visualización conceptual que da lugar a contrastar y comprar los estudios revisados.

Con respecto del segundo recurso, el gestor de información usado es Zotero. Este permite ordenar los documentos dentro de una biblioteca en el PC y en la nube que se actualiza de manera constante. Este gestor ayuda a recuperar los metadatos y elaborar informes de las notas agregadas al documento que se registra en la biblioteca. Así mismo ofrece la posibilidad de generar una ficha bibliográfica con los datos destacados recuperados de la matriz.

\section{RESULTADOS CUANTIFICADOS DE LA BÚSQUEDA}

Como parte del análisis bibliométrico se identificaron un total de 86 estudios, dentro de los cuales se contabilizan 60 artículos académicos, 11 libros, 3 capítulos de libro, 2 ensayos, 3 tesis, 1 simposio y 6 resúmenes de conferencias. Respecto a la relevancia, se establecen descriptores que van de alta a nula. Se cuentan con 29 documentos de alta relevancia, 32 con media-alta, 15 se encuentran en categoría media, 4 en media-baja, 4 en baja y 2 en nula relevancia. Cabe mencionar que esta discriminación categorial se establece a partir de las variables establecidas en la metodología (ABP, autonomía, lingüística e interculturalidad) y sus aportaciones significativas para la articulación con la enseñanza aprendizaje de las lenguas extranjeras.

En cuanto a las producciones de investigación científica sobre el tema se observa que Europa y América son los continentes que más exploran este tipo de temáticas. Cabe mencionar que Europa cuenta con $34 \%$ de los documentos encontrados y es punta de lanza en cuanto a la 


\section{EST U D I S Y E N A Y O S}

enseñanza de lenguas extranjeras, siendo Francia y España los países con mayor número de publicaciones. Con respecto a América, se contabilizaron el $41 \%$ de las fuentes, y se observa que la producción científica muestra interés por igualar en un primer momento a Europa, y posteriormente por proponer nuevas visiones y perspectivas. México y Estados Unidos son los países que cuentan con mayor producción. En Asia se encontraron $22 \%$ de investigaciones siendo Malasia el que cuenta con mayor número de investigaciones y en África solo el 3\%, donde se reportan trabajos de Nigeria y Camerún. (Ver gráfico 1).

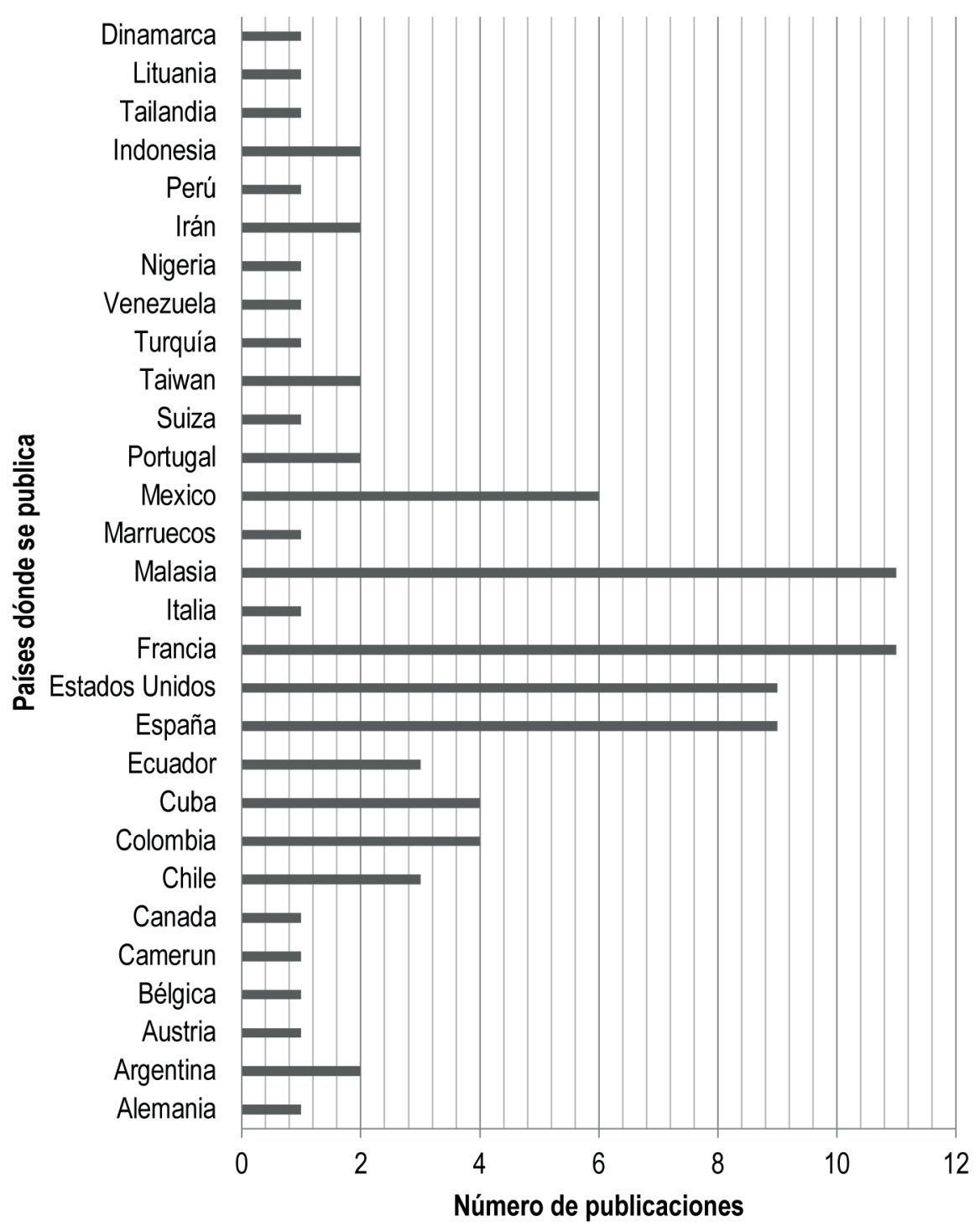

Gráfıco 1. Producciones científicas por países. Nota: Gráfico de elaboración propia

El Gráfico 1 muestra claramente los países punteros en trabajos científicos. Esto permite, por un lado, proponer redes de comunidades científicas para realizar investigaciones de frontera que aporten nuevas visiones al acercamiento de la temática; y por otro, visualizar las producciones científicas en las temáticas para poder tener un mejor acceso a la información, incrementar la participación e identificar la variedad de investigaciones en la temática que nos atañe. 


\section{ESTUDIOS Y ENSAYOS}

Con el fin de establecer un mapeo temporal sobre las producciones científicas sobre la temática en cuestión se consideran las últimas décadas. El punto de arranque son los años ochenta, pues es donde se gesta y empieza a aplicar el enfoque comunicativo, la competencia lingüística y el ABP. Como se aprecia en el Gráfico 2, se agrupan las publicaciones realizadas entre 1980 y 2000, así como entre 2002 y 2007. Es a partir de la última década se muestran las publicaciones por año con la finalidad de conocer las tendencias. Como se puede observar los años de producción más fructíferos son el 2017 y 2018. A partir de estos datos, se puede concluir que la temática ha tomado impulso en los últimos años, por lo que es estudiada aportando nuevos elementos al campo de conocimiento. (Ver Gráfico 2).

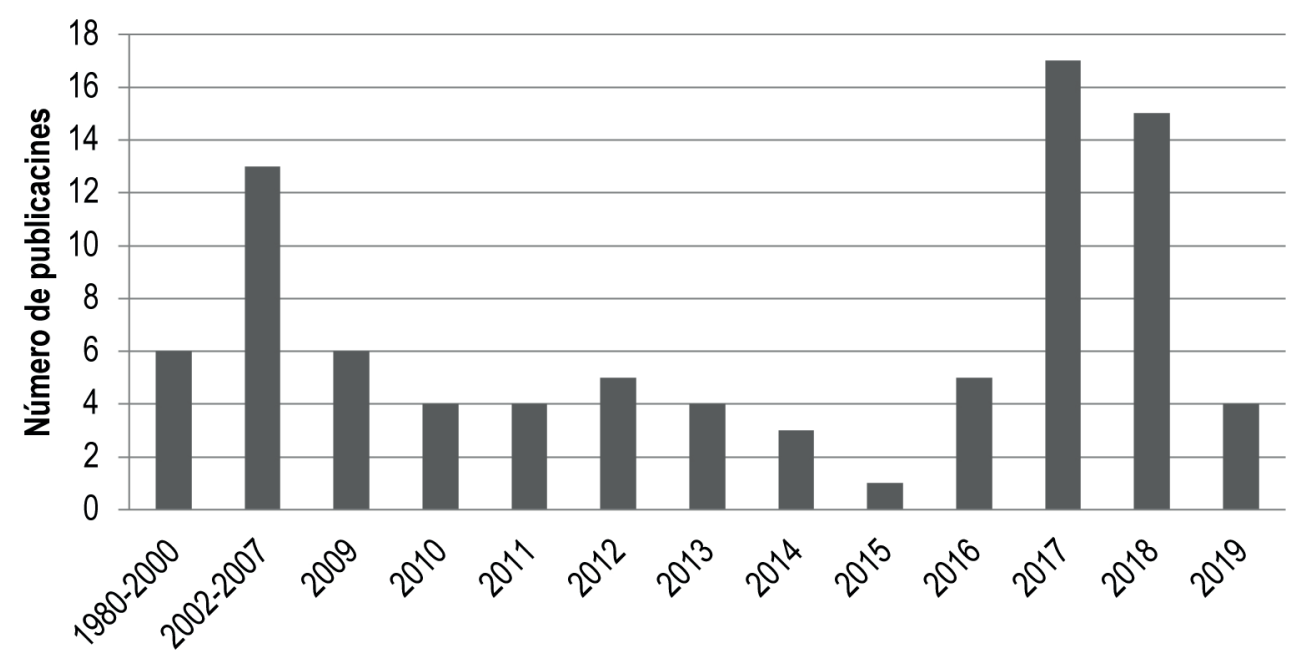

Año de publicación

Gráfıco 2. Fuentes por año de publicación. Nota: Gráfico de elaboración propia

Respecto de la metodología se observa que los estudios realizados guardan un cierto equilibrio entre los cualitativos, los cuantitativos, los mixtos y los que implican meta-análisis y revisión de literatura. Esta clase de estudios representan el 68\% del total de las fuentes, dentro de las que se contabilizan $13 \%$ como estudios cualitativos, $21 \%$ como cuantitativos, $12 \%$ como mixtos y el $22 \%$ más que como estudios, son vistos como documentos de meta-análisis llegando a establecer sus conclusiones como posturas teóricas y conceptuales, sin ofrecer realmente un panorama más específico o concluyente de estudios empíricos. La mayor carga analítica es dejada al lector, quien podrá utilizar o no las posturas planteadas en su práctica.

Generalmente la temática estudiada en las investigaciones cualitativas, cuantitativas y mixtas; es el ABP con $16.8 \%$ la metodología de enseñanza de lenguas extranjeras con $11.6 \%$, seguido de la autonomía con $6.9 \%$ y la interculturalidad con $5.8 \%$. Los estudios en su mayoría se observan de manera independiente, sin embargo, existen algunos articulados entre sí de forma pareada; por ejemplo: estudios de lengua e interculturalidad, autonomía y metodología de enseñanza, ABP y lenguas extranjeras, ABP y autonomía.

En el caso de las investigaciones cualitativas buscan describir las percepciones de los estudiantes sobre esta estrategia y su impacto en el aprendizaje de lenguas, tomando en cuenta el desarrollo de habilidades lingüísticas y aprendizaje autónomo (Badrinathan, 2017). 
En el caso de las investigaciones cuantitativas se miden los resultados y no el proceso de aprendizaje haciendo principal énfasis en el desarrollo de habilidades productivas orales y escritas; y comprensión de textos (Mohammadi, 2017; Zuriya, Agustina y Falarina, 2018). De manera indirecta se observa el aspecto lingüístico, no obstante, no se encontraron evidencias de este tipo de investigaciones que traten a la interculturalidad como un aspecto mesurable.

En cuanto a las investigaciones mixtas se centran en conocer las percepciones de los estudiantes y sus efectos en el desarrollo de la escritura y formación profesional (Aliyu, 2016, 2017; Aryanti y Artini, 2017; Fard y Vakili, 2018; Rashid et al. 2016), considerando indirectamente el aprendizaje autónomo. En el vínculo con la interculturalidad las investigaciones mixtas se concentran en analizar el impacto en las actitudes de los estudiantes respecto de su identidad como miembros de una cultural.

De manera general los hallazgos muestran múltiples vertientes. En el caso de la interculturalidad se fundamentan en la formación docente como parte esencial para promover la cultura en las aulas (Moya-Chávez, Moreno-García y Núñez-Camacho, 2018). Se destaca que las representaciones sociales que se puedan tener de las personas, sus hábitos y modos de ser son primordiales para el proceso hermenéutico de la lengua trasladado a los actos de habla de los usuarios o hablantes (Abdellaouy, 2018; Bizarro, 2018).

De este planteamiento no sorprende que la interculturalidad se halle al interior en la enseñanza aprendizaje, en especial cuando se desarrollan aspectos pragmáticos y socio-lingüísticos, o como lo presentaría el enfoque comunicativo y la perspectiva accional, cuando se llevan a cabo las tareas asignadas. Así mismo el rol de los participantes de este proceso, docentes y estudiantes, es de apertura y reconocimiento del otro y de sí mismo (Abdellaouy, 2018), pues en la medida en que mejor se aborden los temas culturales lingüísticos, existirá una mayor adquisición significativa, y no sólo transmisión de datos que se aprenderán de memoria, sin dotarlos de una relación y un significado particular.

Respecto del tema de aprendizaje autónomo se observa que en la autonomía que se desarro1la en las lenguas extranjeras interviene, además de la reflexión y el pensamiento crítico para la toma de decisiones, el aspecto lingüístico. En los estudios realizados destacan aquellos que lo relacionan de manera evidente con los centros de auto-acceso (Velázquez, Rodríguez y Nieves, 2018; Velázquez y Santiesteban, 2019), los cuales están diseñados para permitir que el estudiante decida los materiales con los que trabajará la lengua para desarrollar las habilidades productivas (expresión oral y escrita) y receptivas (comprensión oral y escrita) a partir del diagnóstico, las estrategias de aprendizaje, los resultados en las actividades y la auto-evaluación (Flores y Meléndez, 2017). Así mismo también los estudios que se centran en estrategias relacionadas con actividades situadas donde se lleva a cabo una mayor acción-interacción por parte del estudiante.

En la autonomía lingüística ocurre un proceso paulatino y consciente en el proceso formativo y de aprendizaje. No obstante, el problema que se observa en todos los casos estudiados es la concepción e imagen que se tiene de un individuo autónomo (Molina et al., 2018). En las creencias, los docentes consideran que los estudiantes no tienen tan desarrollado este rasgo y esperan mucho de ellos, mientras que los estudiantes se ven a sí mismos como personas independientes capaces de tomar decisiones y ejecutar actividades y tareas sin recurrir al docente. 
Ahora bien, el ABP se trata de una estrategia centrada en el estudiante y en los contenidos bajo una visión de aprendizaje activo donde se vehicula la sistematización de la información, el desarrollo de la autonomía y el aprendizaje de idiomas en aspectos particulares de la lingüística y las competencias comunicativas. Todas refieren resultados alentadores y favorecedores, haciendo de ella una estrategia posible de emplear en cualquier ámbito académico, adaptando los problemas a situaciones posibles de suceder.

En el caso del estudio de roles, que existe una sinergia entre ambos participantes. Por un lado, el estudiante es visto como un ser social activo que participa de su formación de manera semiindependiente (Ansarian y Mohammadi, 2018; Aliyu, 2017; Aryanti y Artini, 2017). Por otro lado, el docente a pesar de tener un rol pasivo en el aula, no desaparece. Al contrario, su labor es la de guiar a los estudiantes para lograr mejores investigaciones y así proporcionar una propuesta de resolución favorable (Lin, 2017). Esto implica necesariamente un proceso de aprendizaje autónomo, por lo que es favorable, en un primer plano, en el aprendizaje de aspectos lingüísticos y, en un segundo plano, en el desarrollo de la competencia intercultural; los cuales se consideran en esta investigación.

En cuanto a la explotación de la estrategia, ha sido utilizada mayormente para mejorar y potenciar la comprensión lectora (Lin, 2017), la expresión escrita (Aliyu, 2017), explotar el aprendizaje y fijación de vocabulario (Ansarian y Mohammadi, 2018) y desarrollar la expresión oral (Zuhriyah, Agustina, y Fajarina, 2018).

De manera particular, se hallan dos investigaciones que combinan el ABP con otra estrategia dando resultados favorables en el desarrollo de las lenguas extranjeras y aprendizajes de contenido. Rillero et al. (2017) lo combinaron con el término english language learning, dando como resultado PBELL (Problem-Based-Enheaced-Language Learning), el mismo que se implementó a 26 escuelas públicas impactando favorablemente a casi 2000 estudiantes de origen migrante. Por su parte, Mohd-Ali et al. (2018) proponen articular el ABP con LcCRAFT (Language Case Crafting). Dicha articulación permite elaborar casos para desarrollar la lengua extranjera per se y contenidos de otras asignaturas impartidos en la lengua extranjera.

Pese a estos aspectos positivos, los estudios sobre esta estrategia implementada en el aprendizaje de lenguas cuentan con áreas grises, principalmente dos: el rol del docente como guía (Lin, 2017; Mohd et al., 2018; Muñoz, 2018; Travieso y Ortiz, 2018) y los aspectos culturales (Ansarian y Mahammadi, 2018; Lin, 2017). Esto permite identificar las vetas que son posibles de ser exploradas y dar mayor luz a la implementación, adaptación y aplicación del ABP como una estrategia que posibilita el desarrollo de la competencia comunicativa y de la autonomía.

Los instrumentos empleados son la encuesta, bitácora de observación, entrevistas semi estructuradas y cuestionarios, pre-test y post-test, así como actividades escritas y rúbricas de dichas actividades. Cada uno de ellos es justificado por el tipo de investigación que realiza, de tal manera que puedan dar luz a los objetivos que las investigaciones planean. Los primeros son mayormente empleados en las investigaciones cualitativas, ya que abordan las concepciones que tienen los sujetos sobre el objeto de estudio. Los últimos son empleados en investigaciones cuantitativas, pues recuperan en su mayoría datos estadísticos sobre el abordaje del objeto de estudio. 
En cuanto a la población y muestra, la mayoría de los participantes son estudiantes en la educación superior que se encuentran cursando clases de lenguas extranjeras en distintos niveles y contextos universitarios como lo son universidades públicas y privadas, formación de profesores de lenguas extranjeras y cursos de idiomas generales. Sin embargo, algunas de las investigaciones, sobre todo las cualitativas, se llevan a cabo con docentes de lenguas extranjeras. Finalmente, en una de ellas con inmigrantes que están en proceso de aprender la lengua del país de acogida.

Los hallazgos recogidos desde el marco epistemológico - teórico, muestran dos ejes centrales, el constructivismo y el cognitivismo. De estos se desprenden sus enfoques, teniendo que en el constructivismo se decanta por el sociocultural y el socio constructivismo. Las razones se observan en los objetivos de las investigaciones, pues buscan identificar los conocimientos que los estudiantes construyen de manera social y participativa con el resto de los compañeros, por lo que se estudian desde una mirada inter-subjetiva.

Dentro de los autores que más se mencionan está Vygotsky, directamente desde el aspecto constructivista social pues es quien propone esta línea teórica. También se encuentran a Puren (2014) y Bourguignon (2006) quienes trastocan aspectos de aprendizaje activo y perspectiva accional particularmente en la enseñanza del francés como lengua extranjera, recuperado la gramática, léxico, pronunciación y pragmática (Abdellaouy, 2018; Ansarian y Mohammadi, 2018; Lin, 2017). Esta teoría argumenta las investigaciones sobre interculturalidad y particularmente el aprendizaje de las lenguas extranjeras como un proceso y producto social.

Por su parte el cognitivismo se dirige a estudios de meta-cognición y psicología educativa donde se analizan perspectivas, impactos y desarrollo de aprendizajes en los estudiantes desde una mirada intra-subjetiva. Las actividades que más se desarrollan en las investigaciones están ligadas al proceso de aprendizaje y conocimientos meta-cognitivos que son presentados por Aliyu (2017), Ansarian y Mohammadi (2017) y Flores y Meléndez (2017) quienes realizan investigaciones centradas en el desarrollo del pensamiento crítico y toma de decisiones. Particularmente esta teoría soporta los estudios sobre el ABP, aunque algunos otros apoyan el proceso de construcción del lenguaje al interior del sujeto.

Así mismo, también se hallan estudios dónde el constructivismo y el cognitivismo convergen. Por un lado, dan cuenta el proceso interno del estudiante para lograr aprendizajes y cómo son capaces de recuperar la información, procesarla y emplearlas en situaciones concretas. Por otro lado, describen el proceso social en el que dichos conocimientos son empleados, los contextos y las habilidades de los estudiantes respecto de las lenguas extranjeras (Rillero et al., 2017). En este sentido, las investigaciones buscan analizar perspectivas, impacto y motivación de los estudiantes y docentes en las clases de lenguas extranjeras. Algunos otros estudios no ponen de manifiesto de manera explícita la teoría en la que se gesta el conocimiento, no obstante, es posible apreciarla (s) a partir de los objetivos establecidos que en todos los casos se circunscriben en el constructivismo y en el cognitivismo.

\section{DISCUSIÓN}

A partir de la revisión de literatura se puede afirmar que los estudios realizados sobre el aprendizaje de las lenguas extranjeras se han llevado a cabo durante varios años y han empleado di- 
versas estrategias para que se logre, esto tomando en cuenta las metodologías, los enfoques y las didácticas que permiten la adaptación a los distintos contextos en donde se lleva a cabo (Martín, 2010). Así mismo, se identifican como principales teorías que la soportan a la cognitiva y la social constructivista.

En todos los estudios se constata que existe una vinculación directa con la autonomía, el pensamiento crítico y la resolución de problemas, con la investigación de por medio. De igual manera, se observa que su aplicación favorece estos aspectos y más el aprendizaje de lenguas extranjeras. Como aspecto importante a destacar es la falta de vinculación del ABP con aspectos culturales de las lenguas extranjeras. Se puede asumir que está dado por entendido, ya que la cultura y la lengua van de la mano, sin embargo, hay un vacío en estudios donde se ponga de relieve la interculturalidad usando este tipo de estrategia.

Respecto de la interculturalidad, la gran parte de las investigaciones exponen que la lengua y la cultura son indisociables, pues forman parte de la formación integral de la persona además de considerarla una característica que dota de identidad a los individuos (Abdellaouy, 2018; Bizarro, 2018, Doria, 2017; Herrera y Ortiz, 2018; Moya-Chávez, Moreno-García y Núñez-Camacho, 2019; Sumonte, et al., 2019). La lengua es cultura y viceversa. Ambas aportan entre sí un valor simbólico de los que representan y se construyen una a la otra. Por ende, la preocupación por considerar a la cultura un elemento fundamental en el proceso de enseñanza aprendizaje de los idiomas es uno de los rasgos característicos en los estudios.

Actualmente muchos de los métodos presentan la gramática a partir y al interior de un tema cultural, apoyándose en el enfoque comunicativo (Bourguignon, 2006; Puren, 2014). No obstante, esta proposición, es de subrayar que las clases privilegian en su mayoría los aspectos lingüísticos referidos a la norma (gramática, morfosintaxis, ortografía y pronunciación) mediante ejercicios de sistematización, que poco tienen que ver con la manera de usar la lengua en un ambiente natural; dejando en segundo plano la discusión misma del tema en el que es presentada la norma.

Esta situación deja abierta la ventana para realizar estudios que den igual prioridad a la cultura y a la lengua; que permitan descubrir al estudiante la norma de forma deductiva, y que sea capaz de expresar sus gustos, preferencias y opiniones sobre una temática cultural —no necesariamente las artes, pues cultura engloba todas las expresiones de vida de un individuo, por lo que explorar temas como alimentación, desarrollo económico, derechos humanos, entre otros, es pertinente en este contexto- en otros términos, dejar de permanecer ajenos al contexto global y ser participativos de él.

En lo relativo al aprendizaje autónomo es visto como el ejercicio de aprender para conocer lo que supone aprender a aprender y que se manifiesta por el placer de comprender y descubrir, el ejercicio de una curiosidad intelectual que parte de una cultura general a una de especialización; y la práctica del pensamiento concreto y abstracto mediante en proceso deductivo e inductivo (Delors, 1997). Sin embargo, Cabrera (2009) señala que antes de esto, debe de enseñarse a aprender, proveyendo estrategias y herramientas a los estudiantes que les permitan ser autónomos. En cualquiera de los dos casos, donde el punto de partida es diferente, uno es con el docente y otro con el estudiante mismo; la finalidad es la misma, lograr la reflexión y el pensamiento crítico para la toma de decisiones independiente. 
En un sentido más analítico, el proceso de aprendizaje autónomo es guiado por la meta-cognición, en consecuencia, existe una mayor organización y jerarquización de los datos y procesos que ocurren al interior del individuo, dando como resultado una reacción más acertada ante situaciones determinadas. En cualquiera de los casos, la autonomía necesariamente conlleva una actividad consciente del estudiante, donde se responsabiliza de aquello que hace y cómo lo hace. En las aulas se trata de un proceso formativo paulatino y largo, pues inicia en la educación básica y culmina en la educación superior, y su práctica implica la guía por parte del docente.

Los estudios realizados permiten explorar y profundizar diversas posibilidades como las creencias y autoimagen como seres autónomos, los tipos de estrategias que sean efectivas y eficaces en el desarrollo de la independencia, el rol del docente como guía en los aprendizajes y formador de individuos autónomos capaces de tomar decisiones y responsabilizarse por ellas, la motivación misma por querer descubrir y aprender independientemente de la educación (formal, no formal, informal), e incluso darle un giro y verlo a través de la mirada de las inteligencias múltiples, considerando las necesidades y estilos de cada estudiante.

Finalmente, el ABP es una estrategia utilizada a lo largo de los años en distintas disciplinas. Su proceso de investigación, análisis, toma de decisiones, resolución del problema bajo una mirada colaborativa, permite poner en acción aspectos cognitivos y socio-constructivistas (Barrows, 1996). Su éxito o fracaso está determinado por el planteamiento del problema y la claridad de los objetivos a alcanzar, sumado a esto, el rol docente y el rol de los estudiantes también son importantes, pues mientras el primero es guiar el segundo es auto-regular los aprendizajes. Es así que se promueve indudablemente un desarrollo intelectual, científico, cultural y social de todos los participantes.

Respecto de los roles, en la mayoría de la literatura se centran en describir las actividades de los estudiantes y el proceso mismo que conlleva el ABP. Si bien, al tratarse de un aprendizaje activo centrado en el alumno donde es normal que esto suceda, el actuar del docente como instructor, guía, mediador, es vital para que los objetivos se cumplan, y más aún, es fundamental que el docente conozca la estrategia para conducir a los estudiantes a lo largo del proceso permitiendo que existan aprendizajes significativos e independencia. Por su parte, en los aspectos culturales no se reportan estudios que permitan explotarlos de manera expresa y evidente. Esto viene a enfatizar la importancia de crear escenarios en dónde se toquen temas culturales y además se discutan para lograr una formación más integral y una mejor comprensión del otro.

\section{CONCLUSIONES}

La interculturalidad, el aprendizaje autónomo y el ABP no son temas nuevos, pero sí vigentes. Como se ha visto, se han realizado varios estudios en dónde se presentan de manera aislada o articulados entre sí en distintos contextos, se trate de niveles educativos, áreas de estudio, grupos de personas o comunidades, por mencionar las más recurrentes. La importancia de todos ellos es que brindan la posibilidad de indagar en aspectos que no se consideraban relevantes al momento de iniciarlos permitiendo incrementar el acervo científico e innovaciones en las prácticas educativas. 


\section{EST U D OS Y ENSAYOS}

\section{REFERENCIAS}

Abdellaouy, M. (2018). L'ensegniement marocain et la question de l'interculturel: analyse des représentations. Synergies Chili.14. 71-88. https://bit.ly/3dbJUOo

Aliyu, M. M. (2017). A Problem-Based Learning approach to develop metacognition and writing performance of nigerian undergraduates [Tesis doctoral, Universiti Putra Malasya]. Repositorio https://bit. ly/2zBTsmW

Ansarian, L. \& Mohammadi, F. (2018). Problem-based learning in action: Review of empirical studies. Pertanika J. Soc. Sci. \& Hum. 26, 13-26. https://bit.ly/36BQPh5

Aryanti, N. \& Artini, L. (2017). The impact of Problem-based learning on productive skills ans attitude towards english language learning [resumen de ponencia]. 2nd International Conference on Innovative Research Across Disciplines, ICIRAD 2017. DOI https://doi.org/10.2991/icirad-17.2017.4

Badrinathan, V. (2017). Autonomie de l'apprenant en français : quel rôle pour les techniques théâtrales? Revista Synergies, 10, 139-152. https://bit.ly/2Nw4bmu

Barrows, H. (1996). Problem-Bades Learning in medecine and beyond: a brief overview. En Bringing Problem-Based Learning to Higher Education: Theory and Practice. New Directions for Teaching \& Learning S. N., 68, 3-13. https://bit.ly/2TGJk3j

Bizarro, R. (2018). La compétence interculturelle en cours de LE. Quelques réflexions et exemples. Intercâmbio, 2a. Serie, 7, 39-48 https://bit.ly/3ent0w6

Bravo-Cedeño, G., Loor-Rivadeneira, M. y Saldarriga-Zambrano, P. (2017). Las bases psicológicas para el desarrollo del aprendizaje autónomo. Revista científica Dominio de las Ciencias. 3. (1 Especial).32-45 DOI http://doi.org/10.23857/dc.v3i1.368

Bourguignon, C. (2006). De l'approche communicative à l' « approche communic-actionnelle »: une rupture épistémologique en didactique des langues-cultures. Synergies Europe, 1, 58-73. https://bit. ly/36x4mGN

Cabrera, I. (2009). Autonomía en el aprendizaje: direcciones para el desarrollo en la formación profesional. Actualidades Investigativas en Educación, 9 (22).1-22. https://bit.ly/3gATKeM

Consejo de Europa (2002). Marco común europeo de referencia para las lenguas: aprendizaje, enseñanza, evaluación. 2 ed. Anaya

Delors, J. (1997). La educación encierra un tesoro. UNESCO

Doria, P. (2017). Interculturalidad: miradas múltiples. Desafío creativo y cooperativo. Cuadernos del Centro de Estudios de Diseño y Comunicación. 64, (XVIII). 85-97. DOI http://doi.org/10.18682/cdc.vi64

Fard, E. E. \& Vakili, A. (2018). The effect of Problem-based Learning in Iranian EFL learners' vocabulary learning. The Journal of Asia TEFL, 15 (1), 208-216. DOI http://dx.doi.org/10.18823/ asiatefl.2018.15.1.15.208

Flores, L. y Meléndez, C. (2017). Variación de la autonomía en el aprendizaje, en función de la gestión del conocimiento, para disminuir en los alumnos los efectos del aislamiento. RED Revista de Educación a Distancia. 54 (7). DOI http://dx.doi.org/10.6018/red/54/7

Herrera, J. y Ortiz, C. (2018). Interculturalidad en lenguas-culturas extranjeras: un desafío filosófico para América Latina. Cuestiones de filosofía. 4 (22). DOI http://doi.org/10.19053/01235095. v4.n22.2018.8301 


\section{EST U D OS Y ENSAYOS}

Hall, S. y du Gay, P. (2003). Cuestiones de identidad cultural. Argentina: Amorrurtu editores Herrera, J., Ortiz, C. (2018). Interculturalidad en lenguas-culturas extranjeras: un desafío filosófico para América Latina. Cuestiones de Filosofía. 4 (22). 173-199. DOI http://doi.org/10.19053/01235095. v4.n22.2018.8301

Jiménez-Vásquez, M. (2014). El objeto de estudio y el estado del arte. Un proceso interrelacionado de construcción intelectual. En Metodología de la Investigación. UAT.

Jiménez-Vásquez, M. (2009). La construcción del estado del arte. Instituto de Investigaciones sobre la Universidad y la Educación. UNAM.

Lin, L. (2017). Impacts of the Problem-Bases Learning Pedagogy on English Learners' Reading Comprehension, strategy Use and Active Learning Attitudes. Journal of Education and Training Studies. 6 (5). DOI http://doi.org/10.11114/jets.v5i6.2320

López, M. (2008). El Aprendizaje Basado en Problemas, una propuesta en el contexto de la educación superior en México. Tiempo de educar, 9 (18), 109-132. https://bit.ly/2yAAzR3

Martín, M. A. (2010). Apuntes a la historia de la enseñanza de lenguas extranjeras: La enseñanza de la gramática. Tejuelo, 8, 59-76. https://bit.ly/2VpKdxV

Mohammadi, F. (2017). The effect of Authentic Problem-Based vocabulary tasks on vocabulary learning of EFL learners. International Journal of Education \& Literacy Studies, 5 (3), 35-40. DOI http://dx.doi. org/10.7575//aiac.ijels.v.5n.3p.35

Mohd-Ali, S., Harun, H., Ahmad Mahir, N., Massari, N., Mat Saad, N. \& Simkin, K. (2018). Meeting the demands of the 21st century English language learning through PBL-LcCRAFT. GEMA Online Journal of Language Studies, 18 (2), 255-266. DOI http://dx.doi.org/10.17576/gema-2018-1802-17

Molina, T., Quintero, C., Lizcano, C. y Burbano, L. (2018). Creencias y competencias lingüísticas en estudiantes universitarios latinoamericanos ¿son complementarias o se contraponen? Revista Lasallista de Investigación, 15 (2), 246-255. DOI http://dx.doi.org/10.22507/rli.v15n2a19

Morales, P. y Landa, V. (2004). Aprendizaje Basado en Problemas. Theoria, 13, 145-153. https://bit. ly/3gp6xRl

Morel, M. y Buitrago, L. (2017). El estado del arte/ estado de la técnica y la investigación científica y tecnológica. Portal De La Ciencia, 13.11-16. DOI https://doi.org/10.5377/pc.v13i0.5917

Moya-Chávez, D., Moreno-García, N. \& Núñez-Camacho, V. (2019). Interculturality and language teaching in Colombia: the case of three Teacher Education Programs. Signo y Pensamiento. 37 (73). DOI http://doi.org/10.11144/Javeriana.syp37-73.iltc

Muñoz, D. (2017). Problem-Based Learning: An experiential strategy for english language teacher education in Chile. PROFILE Issues in Teachers' Professional Development, 19 (1). DOI http://dx.doi. org/10.15446/profile.v19n1.53310

Orozco-López, E. (2018). ¿Autonomía educativa o interculturalidad? Una educación alternativa entre los pueblos originarios de Chiapas, México. Revista Colombiana de Educación. 74. 37-61 https://bit. ly/2Ba5ULE

Piteria, L. (2014). Estudio sobre la inclusión del componenete cultural del español como LE en centros de educación secundaria de Islandia. (tesis de maestría) https://bit.ly/3d6eqJo

Pretceille, M. (2011). La pédagogie interculturelle: entre multiculturalisme et universalime. LINGVARVM ARENA, 2. 91-101 https://bit.ly/3ekIhxS 


\section{EST U D O S Y E S AYOS}

Puren, C. (2014). La compétence culturelle et ses différentes composantes dans la mise en oeuvre de la perspective actionnelle. Une nouvelle polemique didactique. Intercâmbio. 2a. Serie, 7. 21-38. https:// bit.ly/3d4SKxj

Rashid, R., Mazlan, S., Wahab, Z., Anas, M., Ismail, N., Syed, O. \& Anwar, M. (2016). Problem-based Learning in language education programme: what educator and learners have to say? Man in India Journal, 96 (12), 5315-5322. https://bit.ly/386KRWb

Rillero, P., Koerner, M., Jimenez-Silva, M., Merrit, J. \& Farr, W. (2017). Developing Teacher Competencies for Problem-Based Learning Pedagogy and for Supporting Learning in Language-Minority Students. Interdisciplinary Journal of Problem-Based Learning. 11 (2). DOI http://doi.org/10.7771/1541-5015.1675

Rivadeneira, E. y Bustillos, R. (2017). Aprendizaje basado en la investigación en el trabajo autónomo y en equipo. Negotium, 13 (18). https://bit.ly/3eq6frF

Sumonte, V., Friz, M., Sanhueza, S. yTravieso Morales, K. (2019). Programa de integración lingüística y cultural: migración no hispanohablante. Alpha, 48. 179-193. DOI https://doi.org/10.32735/S0718$\underline{2201201900048625}$

Travieso, D. y Ortiz, T. (2018). Aprendizaje Basado en Problemas y enseñanza por proyectos: alternativas diferentes para enseñar. Revista Cubana de Educación Superior, 1, 124-133. https://bit.ly/31lHaKX

UNESCO (2020). Tesauro de la UNESCO. https://bit.ly/2ZOiQ3B

Velázquez, Y., Rodríguez, Y. y Nieves, O. (2018). El aprendizaje autónomo de las lenguas extranjeras y el uso de las tecnologías de la información y las comunicaciones. Opuntia Brava.10, (1). DOI http://doi. org/10.35195/ob.v10i1.59

Velázquez, Y. y Santiesteban, E. (2019). Relación dialéctica entre la metacognición y la autonomía en el aprendizaje de los profesores en formación de lenguas extranjeras. Opuntia Brava. 11. (OMonográfico especial). DOI http://doi.org/10.35195/ob.v11iEspecial.687

Zaker, A. (2016). The acculturation model of second language acquisition: inspecting weaknesses and strengths. Indonesian EFL Journal. 2, 80-88. DOI http://doi.org/10.25134/ieflj.v2i2.640 